\title{
Periodic orbits of planar integrable birational maps.
}

\section{Víctor Mañosa*}

\author{
Departament de Matemàtica Aplicada III \\ Control, Dynamics and Applications Group (CoDALab) \\ Universitat Politècnica de Catalunya.
}

NOMA'13 International Workshop on Nonlinear Maps and their Applications

September 2013, Zaragoza, Spain.

A general approach, with examples from works with

G. Bastien, A. Cima, I. Gálvez, A. Gasull, M. Rogalski, X. Xarles.

* Supported by Spanish Ministry of Economy and Competitiveness grant DPI2011-25822 and SGR program. 


\section{INTRODUCTION}

We are interested in the study of periodic orbits of planar integrable birational maps. That is

- Planar rational: $F: \mathcal{U} \subseteq \mathbb{R}^{2} \longrightarrow \mathcal{U}$ where $F(x, y)=\left(\frac{f_{1}}{f_{2}}, \frac{f_{3}}{f_{4}}\right)$ where $f_{i}$ are polynomials.

- Birational: The inverse map $F^{-1}$ is also rational.

- Integrable: There is a function $V$ which is a first integral of $F$ in $\mathcal{U}$. That is if

$$
V(F(x, y))=V(x, y) \text {, for all }(x, y) \text { in } \mathcal{U} \text {. }
$$

In addition, we will consider that $V$ is a rational function

$$
V(x, y)=\frac{P(x, y)}{Q(x, y)} .
$$

So the map preserves a foliation of the plane given by algebraic curves

$$
\mathcal{F}_{h}=\{P(x, y)-h Q(x, y)=0, h \in \operatorname{Im}(V)\} .
$$

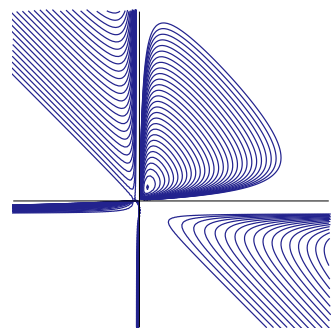




\section{Examples}

They appear in Number theory and Algebraic Geometry as automorphisms of algebraic curves.

The Lyness map $F_{a}(x, y)=\left(y, \frac{a+y}{x}\right)$ associated to $x_{n+2}=\frac{a+x_{n+1}}{x_{n}}$.

It has the with first integral

$$
V(x, y)=\frac{(x+1)(y+1)(x+y+a)}{x y} .
$$

So it preserves the foliation given by

$$
\mathcal{F}_{h}:=\{(x+1)(y+1)(x+y+a)-h x y=0\}
$$

Among a large literature, see for instance Barbeau et al. 1995; Bastien \& Rogalski, 2004a; Beukers \& Cushman, 1998; Duistermaat, 2010; Esch \& Rogers, 2001; Zeeman, 1996. 
They appear as reduction of differential-difference soliton equations. For instance QRT maps, introduced in Quispel et al. 1989, see also Duistermaat, 2010.

\section{D $\Delta$ Nonlinear Schrödinger equation.}

Soliton equation: $\quad-i \frac{d}{d t} u_{n}=u_{n+1}-2 u_{n}+u_{n-1}+\frac{1}{2}\left|u_{n}\right|^{2}\left(u_{n+1}+u_{n-1}\right)$

Reduction: $\quad u_{n}(t)=x_{n} \exp (i \omega t)$

Map:

$F(x, y)=\left(y,-x+\frac{(\omega+2) y}{1+\frac{1}{2} y^{2}}\right)$ a type of Gumovski-Mira map, also in the Mc Millan family of maps, and QRT.

First integral

$$
\begin{aligned}
& V(x, y)=x^{2} y^{2}-2(\omega+2) x y+2 x^{2}+2 y^{2} \\
& \Leftrightarrow \mathcal{F}_{h}:=\left\{x^{2} y^{2}-2(\omega+2) x y+2 x^{2}+2 y^{2}-h=0\right\}
\end{aligned}
$$


Are there planar integrable birational maps with nonrational first integrals?

$$
F(x, y)=\left(\frac{x\left(a+y+d a y+b x+d y^{2}+c x y\right)}{(a+y)(b x+a+y)}, \frac{\left(a b e x+a d x y+b e x y+c x^{2} y+d x y^{2}+a^{2} e+2 a e y+b x^{2}+e y^{2}+a x+x y\right) y}{(c x y+b x+a+y)(b x+a+y)}\right)
$$

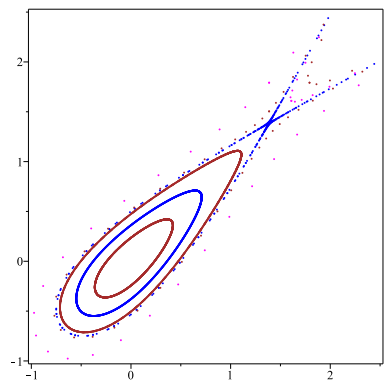

The map is associated to 5-periodic Lyness difference equations

$$
x_{n+2}=\frac{a_{n}+x_{n+1}}{x_{n}}, \text { with } a_{n}=\{a, b, c, d, e\} .
$$

They are non rationally integrable, in fact non meromorphically integrable, for a generic set of parameters Cima et al. 2013ab, although numerically show integrable "features". 


\section{Our objective:}

To show the relationship between the algebraic-geometric properties of the invariant foliation $\mathcal{F}$ and the dynamics of the map $F$.

More precisely, we will focus on the case that $\mathcal{F}$ is generically elliptic.

\section{The topics:}

- The locus of periodic orbits.

- Existence of rational periodic orbits.

- The rotation number function and the set of periods of a map or a family.

- And two short digressions.

\section{The examples (our results):}

- The Lyness Map $F_{a}(x, y)=\left(y, \frac{a+y}{x}\right)$. Gasull et al. 2012.

- The composition map $F_{b, a}(x, y):=\left(F_{b} \circ F_{a}\right)(x, y)=\left(\frac{a+y}{x}, \frac{a+b x+y}{x y}\right)$ associated to 2-periodic Lyness recurrences. Bastien et al. 2013. 


\section{A FIRST DYNAMICAL RESULT: RESTRICTION TO GENUS 0 AND 1 CASES}

A natural phase space to study planar birational maps is

$$
\mathbb{C} P^{2}=\{[x: y: z] \neq[0: 0: 0], x, y, z \in \mathbb{C}\} / \sim
$$

where $\left[x_{1}: y_{1}: z_{1}\right] \sim\left[x_{2}: y_{2}: z_{2}\right]$ if and only if $\left[x_{1}: y_{1}: z_{1}\right]=\lambda\left[x_{2}: y_{2}: z_{2}\right]$ for $\lambda \neq 0$.

The points $[x: y: 1]$ are called affine points and the points $[x: y: 0]$ are called infinity points.

The Lyness map $F(x, y)=\left(y, \frac{a+y}{x}\right)$ can be seen as the map

$$
\tilde{F}([x: y: z])=\left[x y: a z^{2}+y z: x z\right]
$$

except for the points $[x: 0: 0],[0: y: 0]$ and $[0:-a: 1]$.

The invariant real planar foliation $C_{h}=\{(x+1)(y+1)(x+y+a)-h x y=0\}$ is now

$$
\widetilde{\mathcal{C}_{h}}:=\{(x+z)(y+z)(x+y+a z)-h x y z=0\},
$$


Algebraic curves in $\mathbb{C} P^{2}$ are Riemann surfaces and are characterized by their genus.

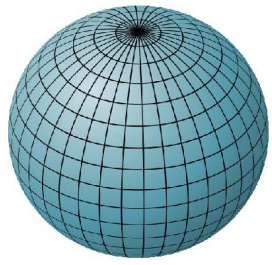

$g=0$

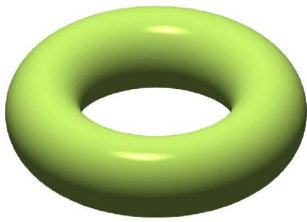

$g=1$,

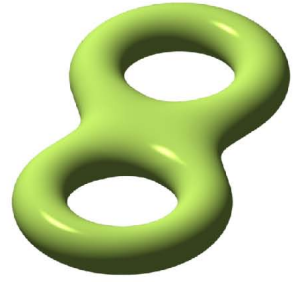

$g=2$

The degree-genus formula states that for a given irreducible curve $C_{h} \in \mathcal{F}$ :

$$
g=\frac{(d-1)(d-2)}{2}-\sum_{p \in \operatorname{Sing}\left(C_{h}\right)} \frac{m_{p}\left(m_{p}-1\right)}{2}
$$

For instance the Lyness' Foliation

$$
\widetilde{\mathcal{F}_{h}}:=\{(x+z)(y+z)(x+y+a z)-h x y z=0\},
$$

has degree $d=3$, so generically the curves have genus $g=1$, and the singular curves have genus $g=0$. 
If our map is NOT Globally periodic, $\mathcal{F}$ is a genus 0 or 1 -foliation.

\section{Proposition.}

A birational map in $\mathcal{U} \subseteq \mathbb{K}^{2}$ with a rational first integral $V$, such that the curves $\{V=c\}$ are non-singular with with genus $g>1$, must be globally periodic. 
If our map is NOT Globally periodic, $\mathcal{F}$ is a genus 0 or 1 -foliation.

\section{Proposition.}

A birational map in $\mathcal{U} \subseteq \mathbb{K}^{2}$ with a rational first integral $V$, such that the curves $\{V=c\}$ are non-singular with with genus $g>1$, must be globally periodic.

Proof:

- If the curves $\{V=c\}$ have generically genus $g>1$ then there exists an open set $\mathcal{V} \subseteq \mathcal{U}$ foliated by curves of these type.

- By Hurwitz Theorem on each of these curves the map must be periodic.

\section{Theorem (Hurwitz, 1893)}

The group of birational maps on a non-singular algebraic curve in $\mathbb{K}^{2}$ of genus $g>1$ is finite and of order at most $84(g-1)$.

- So $F$ is pointwise periodic on $\mathcal{V}$, and hence by the Montgomery Theorem $F$ must be globally periodic on $\mathcal{V}$.

\section{Theorem (Montgomery, 1937)}

Pointwise periodic homeomorphisms in connected metric spaces are globally periodic.

- Since $F$ is rational then it must be periodic on the whole $\mathbb{K}^{2}$ except at the points where its iterates are not well defined. 


\section{THE GENUS 1 CASE. ELLIPTIC FOLIATIONS}

\section{An elliptic curve is a projective algebraic curve of genus 1 .}

Curves of genus 1 are birationally isomorphic to smooth cubic curves.

In the case that $\mathcal{F}$ is given (generically) by elliptic curves, then the group structure of the elliptic foliation characterizes the dynamics of any birational map preserving it.

Theorem (after Jogia, Roberts, Vivaldi. 2006.)

Any birational map $F$ that preserves an elliptic curve $C_{h}$, can be expressed in terms of the group law of the curve as either

- $F_{\mid C_{h}}: P \mapsto P+Q$ where $Q \in C_{h}$ or

- $F_{\mid C_{h}}: P \mapsto i(P)+Q$ where $i$ (and F) is GP of orders 2,3,4 or 6 .

where + denotes the inner sum of $C_{h}$.

What is the group structure? what is this inner sum? 
Take two points $P$ and $Q$ in a nonsingular cubic $C$

(1) Select a point $\mathcal{O}$ to be the neutral element.

(2) Take the third intersection point $P * Q$.

(3) The point $P+Q$ is defined as $P+Q=\mathcal{O} *(P * Q)$.

$$
(C,+, \mathcal{O}) \text {, is an abelian group. }
$$

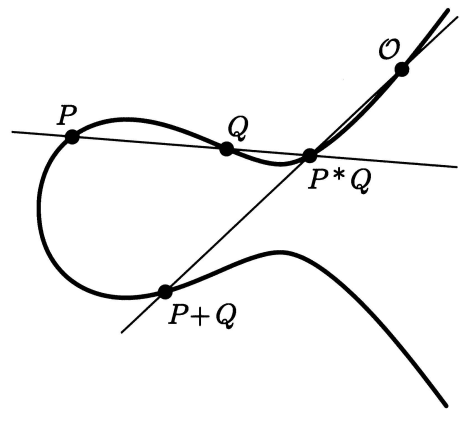

Group law with an affine neutral element $\mathcal{O}$ 
Take two points $P$ and $Q$ in a nonsingular cubic $C$

(1) Select a point $\mathcal{O}$ to be the neutral element.

(2) Take the third intersection point $P * Q$.

(3) The point $P+Q$ is defined as $P+Q=\mathcal{O} *(P * Q)$.

$(C,+, \mathcal{O})$, is an abelian group.
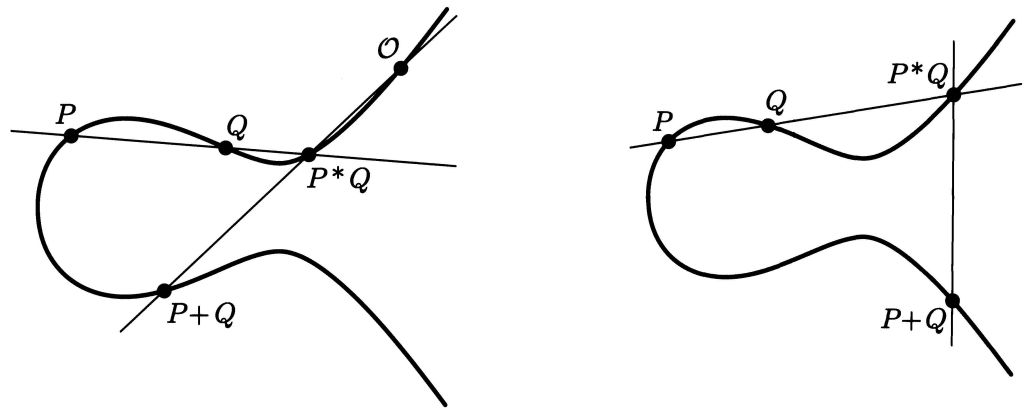

Group law with an affine neutral element $\mathcal{O}$

Group law with $\mathcal{O}=[0: 1: 0]$ 
Theorem (Jogia et al. 2006.)

Any birational map $F$ that preserves an elliptic curve $C_{h}$, there is a choice of $\mathcal{O}$ such that

- $F_{\mid C_{h}}: P \mapsto P+Q$ where $Q \in C_{h}$ or

- $F_{\mid C_{h}}: P \mapsto i(P)+Q$ where $i$ (and F) is GP of orders $2,3,4$ or 6 .

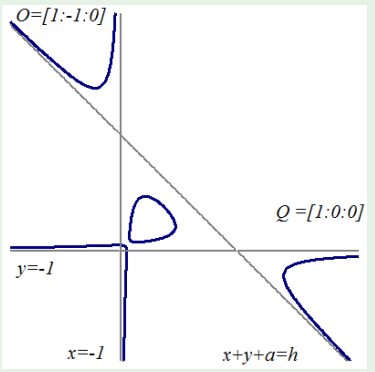

Taking $\mathcal{O}:=[1:-1: 0]$, on each elliptic level the Lyness map is

$$
F(P)=P+[1: 0: 0]
$$

$F_{b, a}(x, y):=\left(\frac{a+y}{x}, \frac{a+b x+y}{x y}\right)$ preserves $C_{h}:=\{(b x+a)(a y+b)(a x+b y+a b)-h x y=0\}$.

Setting $\mathcal{O}:=V=[0: 1: 0]$, on each elliptic level

$$
F_{b, a}(P)=P+[1: 0: 0]
$$


If $F_{\mid C_{h}}: P \mapsto P+Q$, the map is conjugate to a rotation with rotation number $\theta(h)$.

\section{Because}

$$
\left(C_{h}(\mathbb{R}),+\right) \cong\left\{\left(e^{i t}, \pm 1\right) ; t \in[0,2 \pi)\right\} ; \text { with the operation }\left(e^{i t}, v\right) \cdot\left(e^{i s}, w\right)=\left(e^{i(t+s)}, v w\right)
$$

A birational map $F$ on $\left(C_{h},+, \mathcal{O}\right)$ is

$$
F^{\mathrm{n}}: P \longrightarrow P+\mathbf{n} \mathbf{Q}
$$

Hence $C_{h}$ is full of $p$-periodic orbits $\Leftrightarrow p \cdot Q=\mathcal{O}$.

$Q$ is called a torsion point of $C_{h}$.

In summary:

- $Q \in \operatorname{Tor}\left(C_{h}\right) \Rightarrow$ all the orbits in $C_{h}(\mathbb{R})$ are periodic.

- $Q \notin \operatorname{Tor}\left(C_{h}\right) \Rightarrow$ the orbits of $F$ fill densely the connected components of $C_{h}(\mathbb{R})$. 
4. THE LOCUS OF THE PERIODIC ORBITS. Example 1. The Lyness map

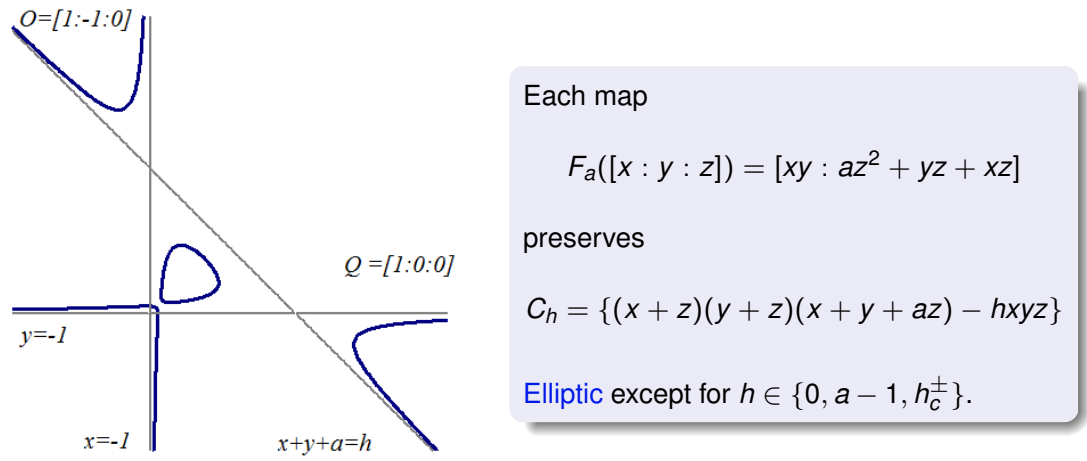

Taking $\mathcal{O}=[1:-1: 0]$ and $Q=[1: 0: 0]$. Observe that both $\mathcal{O}, Q \in C_{h}$ for all $C_{h}$.

$F_{\mid C_{h}}:[x: y: z] \mapsto[x: y: z]+[1: 0: 0] \Leftrightarrow F_{\mid C_{h}}^{\mathrm{n}}:[x: y: z] \mapsto[x: y: z]+\mathbf{n}[1: 0: 0]$

Observe that there exists a $p$-periodic orbit iff $p \cdot[1: 0: 0]=\mathcal{O}=[1:-1: 0]$ 
When $a(a-1) \neq 0$, using the group operation on each elliptic curve, we get

$$
\begin{aligned}
2 Q & =[-1: 0: 1], 3 Q=[0:-a: 1], \\
4 Q & =\left[-a: \frac{a h-a+1}{a-1}: 1\right], \\
\text { and } & \\
-Q & =[0: 1: 0],-2 Q=[0:-1: 1],-3 Q=[-a: 0: 1], \\
-4 Q & =\left[\frac{a h-a+1}{a-1}:-a: 1\right], \\
-5 Q & =\left[\frac{-a^{2}-a h+2 a-1}{a(a-1)}: \frac{a h-a+1}{a-1}: 1\right],
\end{aligned}
$$

- Are there 4 periodic orbits on the elliptic levels? NO

$$
4 Q=\left[-a: \frac{a h-a+1}{a-1}: 1\right] \neq[1:-1: 0]=\mathcal{O} ! !
$$

If $a=1$, then $4 Q=[0: h: 0] \neq[1:-1: 0]=\mathcal{O}$, and no 4-periodic orbits on the genus-0 levels (very easy).

- Are there 9-periodic orbits on the elliptic levels? YES

$$
\begin{gathered}
4 Q=\left[-\mathbf{a}: \frac{a h-a+1}{a-1}: 1\right]=\left[\frac{-\mathbf{a}^{2}-\mathbf{a h}+\mathbf{2 a}-\mathbf{1}}{\mathbf{a}(\mathbf{a}-1)}: \frac{a h-a+1}{a-1}: 1\right]=-5 Q \Leftrightarrow \\
\text { The 9-periodic orbits are in } C_{h_{9}} \text { where } h_{9}=\frac{(a-1)\left(a^{2}-a-1\right)}{a}
\end{gathered}
$$




\section{ON RATIONAL PERIODIC ORBITS.}

The group structure of the $\mathcal{F}$ plays a crucial role when searching rational periodic orbits

A rational orbit is an orbit such that all the iterates have rational coordinates. 


\section{ON RATIONAL PERIODIC ORBITS.}

The group structure of the $\mathcal{F}$ plays a crucial role when searching rational periodic orbits

A rational orbit is an orbit such that all the iterates have rational coordinates.

Our motivation was:

Conjecture (Zeeman, 1996; Bastien and Rogalski, 2004a)

There is no rational periodic orbit of period 9 for the Lyness recurrence $x_{n+2}=\frac{a+x_{n+1}}{x_{n}}$.

Where are the periodic orbits for birational maps on elliptic curves?

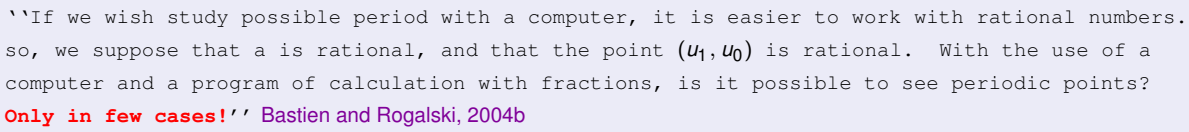


Our maps $F$ restricted to $C_{h}$ can be interpreted as a smooth one-parameter family of diffeomorphism of $\mathbb{S}^{1}$. Thus generically the graph of the rotation function $\theta(h)$ has a devil's staircase shape:

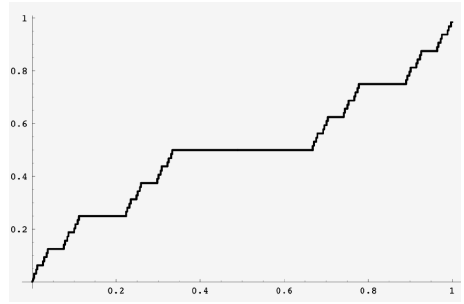

The existence of an open interval $I_{m}$ where $\theta(h) \equiv q / p$, is a consequence of the existence of a hyperbolic $p$-periodic orbit.

$\Downarrow$

Generically, periodic orbits are numerically visible.

In our case $\theta(h)$ is piecewise analytic, but even when $\theta(h)$ is analytic if we have NOT a birational map on elliptic curves you see periodic orbits, e.g. proper Poncelet maps. 
But even when $\theta(h)$ is analytic we find P.O.: playing with a proper Poncelet map.
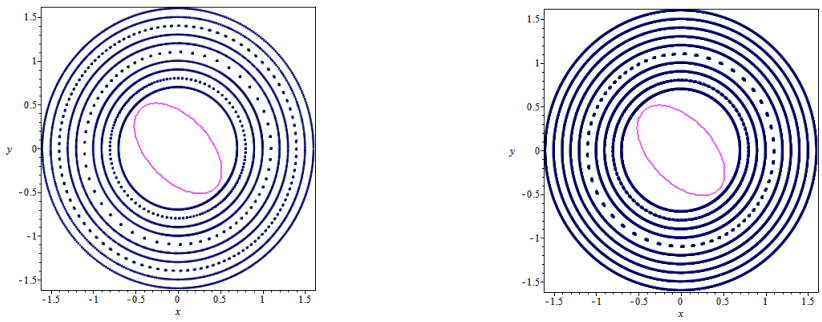

1000 iterates of a Poncelet map
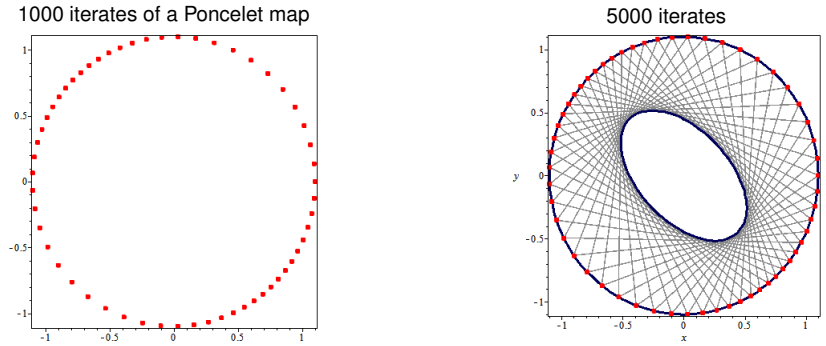

A 57-periodic orbit after 20000 iterates

The Poncelet process

Poncelet Maps are not birational, but using Lie symmetries $\theta(h)$ is analytic, Cima et al. 2010 
The points with rational coordinates in $\left(C_{h},+, \mathcal{O}\right)$ form a subgroup denoted by $C_{h}(\mathbb{Q})$.

Theorem (Mordell, $1922+$ Mazur, 1978)

If $E$ is a non-singular cubic, then $(E(\mathbb{Q}),+)$ is either the finitely-generated abelian group

$$
E(\mathbb{Q}) \cong \mathbb{Z} \oplus \cdots \oplus \mathbb{Z}
$$

or

$$
E(\mathbb{Q}) \cong \mathbb{Z} \oplus \cdots \oplus \mathbb{Z} \oplus \mathbb{Z} / \mathbf{p} \text { where } 1 \leq p \leq 10 \text { or } \mathbf{p}=12
$$

or

$$
E(\mathbb{Q}) \cong \mathbb{Z} \oplus \cdots \oplus \mathbb{Z} \oplus \mathbb{Z} / \mathbf{2} \oplus \mathbb{Z} / \mathbf{p} \text { where } \mathbf{p}=\mathbf{2 , 4 , 6 , 8}
$$

Remember that $F_{\mid C_{h}}: P \longrightarrow P+Q$ will be periodic $\Leftrightarrow Q \in \operatorname{Tor}\left(C_{h}\right)$

$\Downarrow$

\section{Corollary}

Any birational map on $C_{h}(\mathbb{R})$ only can have rational periodic orbits of periods

$1,2,3,4,5,6,7,8,9,10$, XK, 12.

That's why we "don't see" periodic orbits. 


\section{Theorem}

For any $p \in\{1,2,3,4,5,6,7,8,9,10, \not K, 12\}$ there are $a \in \mathbb{Q}^{+} \cup\{0\}$ and rational initial conditions $x_{0}, x_{1}$ such that the sequence generated by $x_{n+2}=\left(a+x_{n+1}\right) / x_{n}$ is $p$-periodic. Moreover these values of $p$ are the only possible minimal periods for rational initial conditions and $a \in \mathbb{Q}$.

A counterexample to Zeeman's Conjecture: taking $a=7$ and the initial condition $x_{0}=3 / 2$, $x_{1}=5 / 7$, we have

$\frac{3}{2} \longrightarrow \frac{5}{7} \longrightarrow \frac{36}{7} \longrightarrow 17 \longrightarrow \frac{14}{3} \longrightarrow \frac{35}{51} \longrightarrow \frac{28}{17} \longrightarrow \frac{63}{5} \longrightarrow \frac{119}{10} \longrightarrow \frac{3}{2} \longrightarrow \frac{5}{7} \longrightarrow \ldots$ 
In fact, we only need to find one rational 9-periodic point to find an infinite number of them.

\section{Proposition}

If $a \in \mathbb{Q}^{+}$is s.t. $\exists$ an initial condition $\left(x_{0}, x_{1}\right) \in \mathbb{Q}^{+} \times \mathbb{Q}^{+}$s.t. the sequence is 9-periodic, then there infinite rational ones. In fact these points fill densely the elliptic curve $C_{h_{9}}$

$$
(x+1)(y+1)(x+y+a)-\underbrace{\frac{(a-1)\left(a^{2}-a+1\right)}{a}}_{h_{9}} x y=0
$$

Proof: Suppose that we have $a \in \mathbb{Q}^{+}$, such that $Q=[1: 0: 0] \in \operatorname{Tor}\left(C_{h_{9}}\right)(\Leftrightarrow 9$-P.O.)

- If $P=(x, y) \in \mathbb{Q}^{+} \times \mathbb{Q}^{+}$is on the oval of $C_{h_{9}}$ then $(2 k+1) P \Rightarrow$ is also on it.

$$
\text { Notice that }(2 k+1) \cdot P \text { are not iterates of } F \text {. }
$$

- The the points $(2 k+1) P$ are rational and fill densely the oval (i.e. $(2 k+1) P \neq \mathcal{O}$ ) because all 9-torsion points are not in $\mathbb{Q}^{+} \times \mathbb{Q}^{+}$,

$$
\text { Remember that }(E(\mathbb{R}),+) \cong\left\{e^{i t}: t \in[0,2 \pi)\right\} \times\{1,-1\} \text {; with the operation }\left(e^{i t}, v\right) \cdot\left(e^{i s}, w\right)=\left(e^{i(t+s)}, v w\right)
$$




\section{Theorem}

There are an infinite number of values $a \in \mathbb{Q}^{+}$and initial conditions $x_{0}(a), x_{1}(a) \in \mathbb{Q}^{+}$giving rise to 9-periodic orbits for the Lyness Eq. 


\section{Theorem}

There are an infinite number of values $a \in \mathbb{Q}^{+}$and initial conditions $x_{0}(a), x_{1}(a) \in \mathbb{Q}^{+}$giving rise to 9-periodic orbits for the Lyness Eq.

\section{Proof:}

- We want to find infinitely many points $(x(a), y(a), a) \in\left(\mathbb{Q}^{+} \times \mathbb{Q}^{+} \times \mathbb{Q}^{+}\right) \cap S_{a}$, where

$S_{a}:=\left\{(a ; x, y): a(x+1)(y+1)(x+y+a)-(a-1)\left(a^{2}-a+1\right) x y=0, x>0, y>0, a>a_{*}\right\}$.

and $a_{*}$ is the infimum s.t. $C_{h_{9}}$ has an oval in $\mathbb{R}^{2,+}$.

- The points in $S_{a}$ s.t. $x+y=23 / 4$, are in an elliptic curve isomorphic to

$$
\mathcal{E}: \quad Y^{2}=X^{3}-\frac{1288423179}{71639296} X+\frac{8775405707427}{303177500672} .
$$

- If we find ONE valid rational point $R \in \mathcal{E}$ NOT in the torsion, then $k \cdot R$ gives an infinite number.

Remember that $(E(\mathbb{R}),+) \cong\left\{e^{i t}: t \in[0,2 \pi)\right\} \times\{1,-1\}$; with the operation $\left(e^{i t}, v\right) \cdot\left(e^{i s}, w\right)=\left(e^{i(t+s)}, v w\right)$

- Using MAGMA we found our seed:

$$
R=\left(\frac{18243}{8464}, \frac{81}{184}\right)
$$

- Recovering the values $(x(a), y(a), a)$ corresponding to the points $k R$, we get the result. 
Universality of the Lyness example. Gasull et al. 2012.

\section{Theorem (Lyness normal form)}

The family of elliptic curves $C_{a, h}=\{(x+1)(y+1)(x+y+a)-h x y=0\}$ over any field $\mathbb{K}$ (not of char. 2 or 3 ) together with the points $\mathcal{O}=[1:-1: 0]$ and $Q=[1: 0: 0]$, is the universal family of elliptic curves with a point of order $n, n \geq 5$ (including $n=\infty$ ).

For any elliptic curve $E(\mathbb{K})$ with a point $R$ of order $n \geq 5, \exists$ ! values $a_{(E, R)}, h_{(E, R)} \in \mathbb{K}$ and a unique isomorphism between $E$ and $C_{a_{(E, R)}, h_{(E, R)}}$ sending the zero of $E$ to $\mathcal{O}$ and $R$ to $Q$.

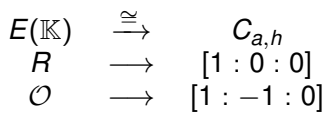

The known results on elliptic curves with a point of order greater than 4 also holds in Lyness curves.

See http://web.math.pmf.unizg.hr/ duje/tors/generic.html 


\section{Proof:}

- Any elliptic curve having a point $R$ that is not a 2 or a 3 torsion point can be brought to the Tate normal form

$$
Y^{2} Z+(1-c) X Y Z-b Y Z^{2}=X^{3}-b X^{2} Z
$$

where $R$ is sent to $(0,0)$.

- The change of variables

$$
X=b z, \quad Y=b c(y+z), \quad Z=c(x+y)+(c+1) z
$$

and the relations

$$
h=-\frac{b}{c^{2}}, \quad a=\frac{c^{2}+c-b}{c^{2}},
$$

show that the curves $C_{a, h}=\{(x+z)(y+z)(x+y+a z)-h x y z=0\}$ and the Tate normal form are equivalent.

- The case $c=0$ corresponds to a curve with a 4-torsion point. 


\section{THE SET OF PERIODS.}

\section{Proposition}

A birational map preserving a foliation of elliptic curves is either rigid or it has an infinite number of periods.

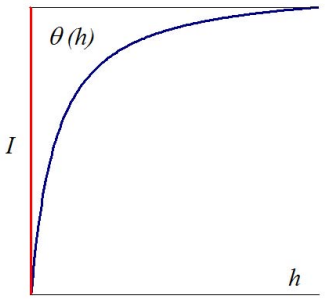

Proof:

- From JRV Theorem on each elliptic curve $C_{h}$ our map is conjugate to a rotation.

- The rotation number function $\theta(h)$ is piecewise continuous for $h \in \operatorname{Im}(V)$.

- Generically $\theta(h)$ is not constant $\Rightarrow \exists$ rotation interval $I$.

For all the irreducible $\frac{q}{p} \in I, \exists$ periodic orbits of $F$ of minimal period $p$.

You can construct $p_{0}$ such that $q / p \in I$ for all $p>p_{0}$, and then check if there is any missing period if $I$ is not optimal. 
Which are the periods of a particular $F$ ? $\Leftrightarrow$ Which are the irreducible fractions in $I$ ?

- It is possible to construct $p_{0}$ s.t. for any $r>p_{0}$ there exists an irreducible fraction $q / r \in I$.

- A finite checking determines for which values of $p \leq p_{0}$ there exists $q / p \in I$.

- Still the forbidden periods must be detected if $I \neq \operatorname{Im}(\theta(h))$.

To construct this value $p_{0}$ we can use, among other methods, this one:

\section{Lemma (Cima et al. 2007)}

Consider a non empty interval $(c, d)$;

Let $p_{1}=2, p_{2}=3, p_{3}, \ldots, p_{n}, \ldots$ be all the prime numbers.

- Let $p_{m+1}$ be the smallest prime number satisfying that $p_{m+1}>\max (3 /(d-c), 2)$,

- Given any prime number $p_{n}, 1 \leq n \leq m$, let $s_{n}$ be the smallest natural number such that $p_{n}^{s_{n}}>4 /(d-c)$.

- Set $p_{0}:=p_{1}^{s_{1}-1} p_{2}^{s_{2}-1} \cdots p_{m}^{s_{m}-1}$.

Then, for any $p>p_{0}$ there exists an irreducible fraction $q / p \in(c, d)$. 
We study the set of periods of the map

$$
F_{b, a}(x, y):=\left(\frac{a+y}{x}, \frac{a+b x+y}{x y}\right)
$$

which preserves the (generically) elliptic curves

$$
C_{h}:=\{(b x+a)(a y+b)(a x+b y+a b)-h x y=0\}
$$

This map appears of the composition map associated to the the 2-periodic Lyness' equations

$$
u_{n+2}=\frac{a_{n}+u_{n+1}}{u_{n}} \text { where } a_{n}=\left\{\begin{array}{lll}
a & \text { for } & n=2 \ell+1, \\
b & \text { for } & n=2 \ell .
\end{array}\right.
$$

Indeed, $F_{b, a}=F_{b} \circ F_{a}$

$$
\left(u_{1}, u_{2}\right) \stackrel{F_{a}}{\longrightarrow}\left(u_{2}, u_{3}\right) \stackrel{F_{b}}{\longrightarrow}\left(u_{3}, u_{4}\right) \stackrel{F_{a}}{\longrightarrow}\left(u_{4}, u_{5}\right) \stackrel{F_{b}}{\longrightarrow}\left(u_{5}, u_{6}\right) \stackrel{F_{a}}{\longrightarrow} \cdots
$$

Setting $\mathcal{O}:=V$, on each elliptic level $\left(C_{h},+, V\right)$ :

$$
F_{b, a}(P)=P+[1: 0: 0]
$$




\section{Theorem.}

Consider the family $F_{b, a}$ with $a, b \in \mathbb{R}$.

(a) If $(a, b) \neq(1,1)$, then $\exists p_{0}(a, b) \in \mathbb{N}$, generically computable, s.t. for any $p>p_{0}(a, b) \exists$ at least a curve $C_{h}$ filled by $p$-periodic orbits.

(b) The set of periods of the family $\left\{F_{b, a}, a, b \in \mathbb{R}\right\}$ contains all minimal periods except 2, 3 .

The set of periods when we restrict ourselves to the case $a, b>0$ and $x, y>0$ contains all minimal periods except 2, 3, 4, 6, 10.

\section{Corollary.}

Consider the 2-periodic Lyness' recurrence for $a, b>0$ and positive initial conditions $u_{1}$ and $u_{2}$.

(a) If $(a, b) \neq(1,1)$, then $\exists p_{0}(a, b) \in \mathbb{N}$, generically computable, s.t. for any $p>p_{0}(a, b) \exists$ continua of initial conditions giving $2 p$-periodic sequences.

(b) The set of minimal periods arising when $(a, b) \in(0, \infty)^{2}$ and positive initial conditions are considered contains all the even numbers except 4, 6, 8, 12, 20.

If $a \neq b$, then it does not appear any odd period, except 1 . 


\section{Proposition}

Fixing $a, b>0 \Rightarrow \exists h_{c}$ such that $\forall h>h_{c} C_{h}$ is elliptic.

The dynamics of $F_{b, a}$ restricted to $C_{h}$ is conjugate to a rotation with rotation number $\theta(h)$.

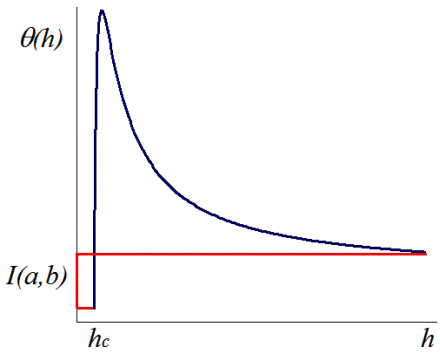

\section{Proposition.}

(a) $\lim _{h \rightarrow+\infty} \theta(h)=\frac{2}{5}$

(b) $\lim _{h \rightarrow h_{c}} \theta(h)=\sigma(a, b)=\frac{1}{2 \pi} \arccos \left(\frac{1}{2}\left[-2+\frac{1}{x_{c} y_{c}}\right]\right)$.

Hence we have, generically, a rotation interval $I(a, b):=\left\langle\sigma(a, b), \frac{2}{5}\right\rangle$.

- (a) is proved using the parametrization of the elliptic curves given by the Weierstrass $\wp$ function as in Bastien et al. 2004a.

- Taking the family $a=b^{2}$ we found that:

$$
\bigcup_{b>0} I\left(b^{2}, b\right)=\left(\frac{1}{3}, \frac{1}{2}\right) \backslash\left\{\frac{2}{5}\right\} \subset \cup_{a>0, b>0} I(a, b) \subset \bigcup_{a>0, b>0} \text { Image }\left(\theta\left(h_{c},+\infty\right)\right) .
$$

- It can be proved that there are $q / p \in(1 / 3,1 / 2)$ with all denominators except $2,3,4,6$ and 10.

- We get the result by studying the energy levels using

$$
p \cdot \mathcal{O}=p \cdot[1: 0: 0]=[0: 1: 0] \text { for } p=2,3,4,6,10 .
$$




$$
\text { What happens if } \mathcal{F}=\{\underbrace{P-h Q=0}_{C_{h}}\} \text { has genus } 0 \text {, or } C_{h} \text { is a genus } 0 \text { curve? }
$$

Each genus 0 curve $C_{h}$ admits a rational parametrization

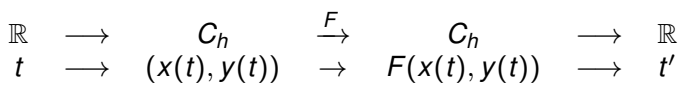

Hence our birational map $F$ on each curve $C_{h}$ admits a representation given by the one-dimensional birational map $t \rightarrow t^{\prime}$.

The only one-dimensional birational maps are the Möbius transformations

$$
t \rightarrow \frac{a t+b}{c t+d}
$$

which are well known. 


\section{References.}

- Barbeau, Gelbord, Tanny; 1995. J. Difference Equations Appl. 1.

- Bastien, Rogalski; 2004a. J. Difference Equations Appl. 10.

- Bastien, Rogalski; 2004b. J. Math. Anal. Appl. 300.

- Bastien, Mañosa, Rogalski; 2013. Int. J. Bifurcations and Chaos. 23.

- Beukers, Cushman; 1998. J. Differential Equations 143.

- Cima, Gasull, Mañosa; 2007. J. Difference Equations Appl. 13.

- Cima, Gasull, Mañosa; 2010. Computers \& Math. with Appl. 60.

- Cima, Gasull, Mañosa; 2013a. Dynamical Systems. To appear.

- Cima, Zafar; 2013b. J. Math. Anal. Appl. To appear.

- Esch, Rogers; 2001 Discrete Comput. Geom. 25.

- Gasull, Mañosa, Xarles; 2012. Discrete and Continuous Dynamical Systems -A. 32.

- Duistermaat; 2010. Discrete Integrable Systems. Springer.

- Jogia, Roberts, Vivaldi; 2006. J. Physics A 39.

- Montgomery; 1937. Amer. J. Math. 59.

- Quispel, Roberts, Thompson; 1988 and 1989. Phys. Lett. A 126. and Phys. D 34.

- Silverman; 2009 The Arithmetic of Elliptic Curves. Springer.

- Silverman, Tate; 1992. Rational Points on Elliptic Curves. Springer.

- Zeeman; 1996. Geometric unfolding of a difference equation. Unpublished paper. 